\title{
Effects of Eight Weelss of Cardiac Rehabilitation Training on Serum Levels of Sirtuin 1 and Functional Capacity of Post- Coronary Artery Bypass Grafting Patients
}

\author{
Nagmeh Alsabah Alavizadeh \\ (PhD) \\ Student of Sports Physiology, \\ Islamic Azad University of \\ Neyshabour, Neyshabour, Iran \\ Amir Rashidlamir (PhD) \\ Department of Sports Physiology, \\ Faculty of Sports Sciences, Ferdowsi \\ University of Mashhad, Mashhad, \\ Iran \\ Seyed Mahmud Hejazi (PhD) \\ Department of Sports Physiology, \\ Faculty of SportsSciences, Islamic \\ Azad University of Mashhad, \\ Mashhad, Iran \\ Corresponding author: Amir \\ Rashidlamir \\ Email: rashidlamir@um.ac.ir \\ Tel: +98-9151514174 \\ Address: Department of Sports \\ Physiology, Faculty of Sports \\ Sciences, Ferdowsi University of \\ Mashhad, Mashhad, Iran \\ Received: 06 Aug 2018 \\ Revised: 02 Oct 2018 \\ Accepted: 06 Oct 2018

\section{(c) (i) (9)} \\ This work is licensed under a Creative \\ Commons Attribution 4.0 License.
}

\begin{abstract}
Background and Objectives: Cardiac rehabilitation improves functional capacity of post-coronary artery bypass grafting (CABG) patients. Therefore, the aim of this study was to evaluate effects of eight weeks of cardiac rehabilitation on serum levels of sirutinl (SIRTl) and functional capacity of post-CABG patients.

Methods: Forty-five non-active men (mean age: $47.4 \pm 3.3$ years, duration of disease: $150 \pm 69.12$ months) were randomly divided into three equal groups: aerobic exercise, combined (resistance and aerobic) exercise and control. The subjects performed trainings at $60-75 \%$ of maximum heart rate, three sessions a week for eight weeks. Blood samples were collected 24 hours before the first exercise session and 48 hours after the last exercise session. Data were analyzed with SPSS 16.0 using one-way ANOVA and the Tukey post hoc test. P-values less than 0.05 were considered statistically significant.

Results: Aerobic and combined exercise increased serum SIRTl levels and functional capacity. There was no significant decrease in body mass index $(\mathrm{P}=0.06)$. The SIRT1 level did not differ significantly between the two exercise groups after the intervention $(\mathrm{P}=0.91)$. There were significant difference between the study groups in terms of the travelled distance $(\mathrm{P}<0.034)$.

Conclusion: Both combined and aerobic exercise can increase serum levels of SIRTl, reduce body mass index and improve functional capacity in CABG patients.

Keywords: Resistance training, exercise, SIRTl.
\end{abstract}

This paper should be cited as: Alsabah Alavizadeh N, Rashidlamir A, Hejazi SM [Effects of Eight Weeks of Cardiac Rehabilitation Training on Serum Levels of Sirtuin1 and Functional Capacity of Post- Coronary Artery Bypass Grafting Patients]. ml.joums. 2019; 13(2):41-47 


\section{INTRODUCTION}

Cardiac rehabilitation is an important component of the comprehensive program on secondary prevention of cardiovascular disease (CVD), which can reduce age-related cardiovascular deaths by about $50 \%$ (1). The rehabilitation program includes physical exercise for improving risk factors such as hyperglycemia, hypertension and obesity (2).

According to the World Health Organization, CVDs such as coronary artery disease cause 17 million deaths per year (3). Post-surgery patients are usually inactive and not rehabilitated. Recent studies show that cardiac rehabilitation with physical exercise can improve exercise capacity, risk factors for coronary artery disease and quality of life (4). Generally, cardiac rehabilitation reduces CVD-related mortalities by $22-25 \%$ (5).

In addition to echocardiography, assessing sirtuin1 (SIRT1) levels has been recently used for diagnosis of heart failure. SIRT1 is a $\mathrm{NAD}^{+}$-dependent deacetylase involved in oxidative stress and endothelial dysfunction, which are associated with endothelial cell pathophysiology and development of CVD, including heart failure (6). Recent studies demonstrated the protective role of SIRT1 in endothelial vascular homeostasis, CVD and other diseases (7-9). SIRT1 ameliorates atherosclerosis and oxidative stress and regulates blood pressure and growth/survival of cardiomyocytes through its deacetylase activity (10). Calorie restriction and physical activity are associated with SIRT1 expression (11). Studies demonstrate that SIRT1 and 3 levels decrease with age, but it is higher in endurance trained individuals than in untrained counterparts (12). Exercise or chronic electrical stimulation (13-15) but not acute exercise $(13,14)$ increase the level of SIRT1 and 3 in the skeletal muscle. SIRT1 expression is high in a healthy heart but decreases following cardiac hypertrophy (16). Although exercise is a major predictor of CVD, the effect of exercise on SIRT1 level of CVD patients is still unknown (17). $\mathrm{Lu}$ et al. demonstrated that low level of SIRT1 in patients with severe heart failure causes oxidative stress and apoptosis (18).

Exercise programs for patients who underwent coronary artery bypass grafting (CABG) are mainly focused on aerobic exercise such as walking (19). A growing body of evidence suggests that resistance training along with endurance training is an effective approach for increasing life span of these patients (20). Decreased muscle mass following CABG has been associated with reduced muscle strength, functional capacity, change of body composition and quality of life (21). Therefore, aerobic endurance training is proposed as part of rehabilitation programs to increase muscle strength and aerobic capacity (22). The six-minute walk test (6MWT) is one of the most useful tools for assessing overall health of CVD patients. The test consists of six minutes of walking on a corridor under complete monitoring of vital signs. It is simpler and more tolerable than other walking tests and better reflects the patient's functional capacity (23). Cardiac rehabilitation has positive effects on risk of death and psychosocial status in patients with CVD (24). Therefore, the present study aimed to study effects of eight weeks of cardiac rehabilitation training on serum levels of SIRT1 and functional capacity of post-CABG patients.

\section{MATERIALS AND METHODS}

Forty-five participants were selected from the Javad-Al-Aeme hospital and randomly divided into three groups: aerobic exercise $(n=15)$, combined exercise $(n=15)$ and control $(n=15)$. Inclusion criteria were age range of 40-60 years, willingness to participate in the study, cognitive health, normal vision and hearing, blood pressure of $<160 / 100 \mathrm{mmHg}$, no history of acute or advanced diseases that could limit exercise ability and inability to use auxiliary devices for walking such as cane and walker. Exclusion criteria included use of medications, occurrence of ventricular arrhythmias, ST elevation or fall during training sessions, respiratory disturbances during rehab and practice and unstable angina. The study was approved by the ethics committee of Islamic Azad University of Neyshabour, Iran (code: IR.IAU.NEYSHABUR.1396, 13).

Subjects in the aerobic and combined exercise groups participated in an 8-week training program based on the recommendations of the American College of Sports Medicine $(25,26)$. The control subjects were asked to carry out basic daily living activities during the study period. All three groups took the 6MWT before and after the exercise program. To perform the test, the patients' vital signs and arterial oxygen saturation were controlled. 
Then, the patient walked on a smooth 30-meter corridor for six minutes. The patients were asked to stop if they were feeling tired. While walking, a trained technician was accompanying the patient and encouraging him/her to continue the activity. In case of acute shortness of breath, chest pain, cyanosis and unwillingness to walk, the test was stopped. Vital signs, arterial oxygen saturation and the covered distance were recorded. The test was performed according to the 6MWT protocol provided by the American Lung Association (27).

One week before the first exercise session, the subjects attended an introductory session to become familiarized with the trainings. The rehabilitation program was carried out three sessions per week for eight weeks. Aerobic training consisted walking on a treadmill for 20-30 minutes, cycling on a fixed bike (10-12 minutes) using the bicycle's ergometer (10 minutes). The subjects warmed-up at the beginning and cooled down at the end of each session with stretching exercises. The trainings began at moderate intensity ( $60 \%$ of maximum heart rate) (28), but the duration and intensity of the trainings gradually increased according to the subjects capacity, so that in the final session the intensity increased to $80 \%$ of maximum heart rate. The exercise intensity was assessed using the Borg effort 6-20 scale (29, 30). Resistance training program included: physio ball squat, shoulder flexion, hip flexion, shoulder abduction, hip abduction, elbow flexion, plantar flexion and ankle dorsiflexion for 8-15 repetitions and three sets. The activities were initially performed with eight repetitions using a weak thera-band (yellow). Then, two repetitions were added to each activity every session until reaching 15 repetitions. The gradual increase of repetitions was repeated after increasing the strength of the thera-band (pink) (26). All activities were performed under supervision of a physician and a CCU nurse. The patients also received psychological and nutritional counseling during the training period. It should be noted that all participants used beta-blockers and angiotensin-antagonists under supervision of a physician.

Blood samples $(10 \mathrm{ml})$ were taken after 10-12 hours of fasting, one day before the first training session and 48 hours after the last training session. Blood samples were incubated for 20 minutes at room temperature and then centrifuged at 3000 RMP for 20 minutes. Serum was separated and stored at $80{ }^{\circ} \mathrm{C}$ until analysis. Serum SIRT1 levels were measured using the Cassabweo ELISA kits with a sensitivity of less than $0.39 \mathrm{ng} / \mathrm{ml}$. Data were analyzed with SPSS 16.0 using one-way ANOVA and the Tukey post hoc test.

\section{RESULTS}

Some characteristics of the participants are presented in table 1.

Pretest serum level of SIRT1 did not differ significantly between the study groups. Mean serum level of SIRT1 increased significantly in both exercise groups, but the posttest level of SIRT1 did not differ significantly between the groups (Table 2). The distance covered in the 6MWT was significantly different between the groups after the intervention $(\mathrm{P}=0.01)$, and the exercise types increased the distance covered similarly ( $\mathrm{P}=0.343)$ (Table 2$)$. The walking frequency and SIRT1 levels increased significantly in both exercise groups after training program.

The results of Tukey post hoc test showed a significant increase in SIRT1 levels after the training period $(\mathrm{P}<0.001)$, but we did not observe any significant difference in the posttest levels of SIRT1 between the aerobic exercise and combined exercise groups $(\mathrm{P}>0.05)$. The walking frequency differed significantly between the study groups $(\mathrm{P}<0.05)$. Moreover, the walking frequency was highest in the combined exercise group (Table 2).

Table 1- Characteristics of the subjects in the exercise groups and the control group

\begin{tabular}{|c|c|c|c|c|c|c|c|}
\hline Group & $\mathbf{N}$ & Age (Year) & Height (m) & $\begin{array}{c}\text { Pretest } \\
\text { bodyweight } \\
\text { (Kg) }\end{array}$ & Posttest body weight (Kg) & $\begin{array}{c}\text { Duration of } \\
\text { disease (months) }\end{array}$ & $\begin{array}{c}\text { Body mass } \\
\text { index }\left(\mathrm{Kg} / \mathrm{m}^{2}\right)\end{array}$ \\
\hline Control & 15 & $47.4 \pm 2.75$ & $170.9 \pm 3.9$ & $80.01 \pm 8.8$ & $79.2 \pm 9.21$ & $149 \pm 69.12$ & $28.22 \pm 2.46$ \\
\hline $\begin{array}{l}\text { Aerobic } \\
\text { exercise }\end{array}$ & 15 & $46.9 \pm 3.23$ & $171.1 \pm 3.6$ & $80.14 \pm 9.12$ & $80.1 \pm 9.3$ & $150 \pm 68.19$ & $22.18 \pm 2.01$ \\
\hline $\begin{array}{l}\text { Combined } \\
\text { exercise }\end{array}$ & 15 & $47.4 \pm 3.23$ & $170.2 \pm 3.5$ & $80.16 \pm 8.99$ & $79.03 \pm 8.95$ & $150 \pm 69.01$ & $26.60 \pm 2.09$ \\
\hline
\end{tabular}


Table 2- Mean serum level of SIRT1 and distance covered in the 6MWT in the study groups

\begin{tabular}{|c|c|c|c|c|c|c|c|c|}
\hline \multirow{3}{*}{$\begin{array}{c}\text { Variable } \\
\text { SIRT1 } \\
\text { (ng/ml) }\end{array}$} & \multirow[t]{2}{*}{ Groups } & \multirow[t]{2}{*}{ Pre-TEST } & \multirow[t]{2}{*}{ Post-TEST } & \multicolumn{2}{|c|}{ K-S } & \multicolumn{2}{|c|}{ ANOVA } & \multirow{2}{*}{$\frac{\text { TUKEY }}{F}$} \\
\hline & & & & $\mathbf{Z}$ & sig & $\mathbf{F}$ & sig & \\
\hline & Control & $0.15 \pm 0.03$ & $* * 0.15 \pm 0.03$ & 0.953 & 0.707 & & & ** $0 / 001$ \\
\hline \multirow{5}{*}{ 6MWT (m) } & Aerobic & $0.16 \pm 0.03$ & $* * 0.25 \pm 0.05$ & 0.865 & 0.088 & $20 / 470$ & $* 0.001$ & 0.915 \\
\hline & Combined & $0.15 \pm 0.3$ & $* * 0.22 \pm 0.4$ & 0.979 & 0.975 & & & \\
\hline & Control & $539 \pm 71.67$ & $* * 547.5 \pm 82.15$ & 0.913 & 0.302 & & $* 0.001$ & \\
\hline & Aerobic & $550.8 \pm 62.54$ & $* * \infty 596.9 \pm 70.8$ & 0.936 & 0.505 & 18.596 & & \\
\hline & Combined & $559.92 \pm \mathbf{3 4 . 5 4}$ & $* *_{\infty} \infty 634.76 \pm 34.87$ & 0.967 & 0.863 & & & \\
\hline
\end{tabular}

significant difference between the control group and the two exercise groups

${ }^{\infty}$ significant difference between the aerobic exercise group and the combined exercise group

\section{DISCUSSION}

The main finding of the present study was the elevated functional capacity in patients with prior $C A B G$ after eight weeks of aerobic and combined exercise. The distance covered in the 6MWT also increased by $8 \%$ in the aerobic exercise group and by $13 \%$ in the combined exercise group.

Continuous aerobic training at moderate intensity is the most commonly used form of cardiac rehabilitation, which increases aerobic power or functional capacity and decreases fat mass in CVD patients (31). Ronau et al. reported that physical activity capacity is the strongest predictor of deaths caused by cardiac events (32). In our study, the distance covered in the 6MWT increased in both exercise groups after the training program, which may indicate that the rehabilitation program significantly increased the patients' physical power. It has been reported that rehabilitation and physical exercise lower CVD-related mortalities by $26 \%$ when compared with cardiac medications (33). Cardiac rehabilitation not only reduces the frequency of medication use (34), but also increases efficiency of open-heart surgery and percutaneous coronary intervention (35). Similar to our study, Mozaffari et al. also found that physical power increases after eight weeks of cardiac rehabilitation (36). Various cardiovascular studies have proven the safety and effectiveness of low- and moderate-intensity aerobic exercise $(32,37)$. In the present study, functional capacity improved in both exercise groups. Improvement of cardiac function via rehabilitation with endurance exercise also increases patients' ability to walk longer distances with less pain and physical problems (38). In a study conducted by Susa et al., nine months of combined exercise was more effective in decreasing body fat percentage and increasing muscle strength in the elderly compared with aerobic exercise (39).

There has been much debate about the most effective intensity, duration and type of exercise $(32,38,40)$. The effects of postCABG exercise on serum SIRT1 level have been mostly investigated in animal models (41). We found that the eight-week exercise program increases SIRT1 level in post-CABG patients. Previous studies suggested that calorie restriction also induces SIRT1 overexpression (42). In line with our findings, Russomanno et al. demonstrated that cardiac rehabilitation could stimulate SIRT1 expression and antioxidant defense system. This is accomplished by increasing the activity of SIRT1 and mitochondrial biogenesis (43). According to histological studies, human atherosclerotic plaques exhibit morphological features of aging. Oxidative stress has been shown to be significantly associated with heart failure and endothelial homeostasis and cellular aging (44). It can be expected that the exercise-induced increase in SIRT1 can also affect health of the cardiac endothelium. Saremi et al. reported improvement of SIRT1 and metabolic indicators in women with type 2 diabetes after eight weeks of aerobic exercise (45). Narou et al. studied the effect of 50 minutes of treadmill running, five days a 
week, for eight weeks on SIRT1, PGC-1 $\alpha$, and AMPK levels in quadriceps muscle of middleaged rats. The results revealed an increase in inflammatory factors and a decrease in anabolic factors with increasing age (46). Scientists believe that during muscle contractions, cAMP-dependent protein kinase and nicotinamide phosphoribosyl transferase increase intracellular $\mathrm{NAD}^{+}$levels, which in turn increases SIRT levels. Finally, SIRT1 is induced by PPAR-alpha and PPAR- gamma and contribute to fat oxidation, glucose uptake and mitochondrial biogenesis (47).

Results of some studies are inconsistent with our findings. For instance, Ma et al. found no change in the SIRT1 level after four weeks of high-intensity interval training at $170 \%$ of peak aerobic power on ergometer (48). In another study, three months of swimming at $70 \%$ of $\mathrm{VO} 2_{\mathrm{Max}}$ did not affect serum level of SIRT in healthy rats despite improving the metabolic status (49). The contradictions between the result of studies may be due to

\section{REFERENCES}

1. Lie I, Arnesen H, Sandvik L, Hamilton G, Bunch EH. Predictors for physical and mental health 6 months after coronary artery bypass grafting. Eur J Cardiovasc Nurs. 2010; 9(4): 238-43. doi: 10.1016/j.ejcnurse.2010.02.001.

2. Aliabad HO, Vafaeinasab M, Morowatisharifabad MA, Afshani SA, Firoozabadi MG, Forouzannia SK. Maintenance of physical activity and exercise capacity after rehabilitation in coronary heart disease: A randomized controlled trial. Glob J Health Sci. 2014; 6(6): 198-208. doi: 10.5539/gjhs.v6n6p198.

3. World Health Organization. Cardiovascular disease. Geneva: WHO; 2008.

4. Quiles J, Miralles-Vicedo B. Secondary prevention strategies for acute coronary syndrome. Rev Esp Cardiol. 2014; 67(10): 844-8. DOI: 10.1016/j.rec.2014.04.013.

5. Mark AW, Ades P, Hamm L, Keteyian S, Lafontaine T, Rotiman J. Clinical evidence for a health benefit from cardiac rehabilitation: an update. Am Heart J (2006); 152(5): 835-841. DOI:10.1016/j.ahj.2006.05.015.

6. Tanno M, Kuno A, Horio Y, Miura T. Emerging beneficial roles of sirtuins in heart failure. Basic Res Cardiol. 2012; 107(4): 273. doi: 10.1007/s00395-0120273-5.

7. Corbi G, Conti V, Russomanno G, Longobardi G, Furgi G, Filippelli A, et al. Adrenergic signaling and oxidative stress: a role for sirtuins? Front Physiol. 2013; 4: 324. doi: 10.3389/fphys.2013.00324.

8. Yu W, Fu YC, Chen CJ, Wang X, Wang W. SIRT1: a novel target to prevent atherosclerosis. J Cell Biochem. 2009; 108(1): 10-3. doi: 10.1002/jcb.22240.

9. Potente M, Dimmeler S. Emerging roles of SIRT1 in vascular endothelial homeostasis. Cell Cycle. 2008; 7(14): 2117-2122. differences in the method of implementation, disease severity and age, gender, body composition and physical readiness of subjects.

\section{CONCLUSION}

Our results indicate that the cardiac rehabilitation training significantly increases serum level of SIRT1 and improves functional capacity in post-CABG patients. Therefore, this type of exercise could be recommended for prevention of coronary artery occlusion after CABG.

\section{ACKNOWLEDGEMENTS}

We would like to express our gratitude to Dr. Alavi for guiding us throughout this research.

\section{CONFLICT OF INTEREST}

The authors declare that there is no conflict of interest.

10. Cui Y, Wang H, Chen H, Pang S, Wang L, Liu D, et al. Genetic analysis of the SIRT1 gene promoter in myocardial infarction. Biochem Biophys Res Commun. 2012; 426(2): 232-6. doi: 10.1016/j.bbrc.2012.08.071.

11. Michan S. Calorie restriction and NAD+ /sirtuin counteract the hallmarks of agine. FRONT Biosci. 2014; 19: 1300-19.

12. Lanza IR, Short DK, Short KR, Raghavakaimal S, Basu R, Joyner MJ, et al. Endurance exercise as a countermeasure for aging. Diabetes. 2008; 57(11): 293342. doi: 10.2337/db08-0349.

13. Gurd BJ, Holloway GP, Yoshida Y, Bonen A. In mammalian muscle, SIRT3 is present in mitochondria and not in the nucleus; and SIRT3 is upregulated by chronic muscle contraction in an adenosine monophosphate-activated protein kinase-independent manner. Metabolism. 2012; 61(5): 733-41. doi: 10.1016/j.metabol.

14. Hokari F, Kawasaki E, Sakai A, Koshinaka K, Sakuma K, Kawanaka K. Muscle contractile activity regulates Sirt3 protein expression in rat skeletal muscles. J Appl Physiol (1985). 2010; 109(2): 332-40. doi: 10.1152/japplphysiol.00335.2009.

15. Palacios OM, Carmona JJ, Michan S, Chen KY, Manabe Y, Ward Iii JL, et al. Diet and exercise signals regulate SIRT3 and activate AMPK and PGC-1 $\alpha$ in skeletal muscle. Aging (Albany NY). 2009; 1(9): 771-83. 16. Ma L, Y Li. SIRT1: role in cardiovascular biology. Clin Chim Acta. 2015; 440: 8-15. doi: 10.1016/j.cca.2014.10.036.

17. Zhang MJ, Zhou Y, Chen L, Wang X, Long CY, Pi $\mathrm{Y}$, et al. SIRT1 improves VSMC functions in atherosclerosis. Prog Biophys Mol Biol. 2016; 121(1):11-5. doi: 10.1016/j.pbiomolbio.2016.02.003. 
18. Lu T-M, Tsai J-Y, Chen Y-C, Huang C-Y, Hsu H-L, Weng C-F, et al. Downregulation of Sirtl as aging change in advanced heart failure.J Biomed Sci. 2014; 21(1): 57. doi: 10.1186/1423-0127-21-57.

19. Passino C, Severino S, Poletti R, Piepoli MF, Mammini C, Clerico A, et al. Aerobic training decreases $B$-type natriuretic peptide expression and adrenergic activation in patients with heart failure. J Am Coll Cardiol. 2006; 47(9): 1835-9.

20. Gaeini A, Sadegh Sattarifard S, CafiZadeh S, Nejatian M. The comparison of eight weeks of combined and aerobic training onfunctional capacity, body composition and strength in post-coronary artery bypass graft cardiac patients. Cardiovascular Nursing Journal. 2013; 2(1): 41-34.

21. Oliveira JL, Galvão CM, Rocha SM. Resistance exercises for health promotion in coronary patients: Evidence of benefits and risks. Int J Evid Based Healthc. 2008; 6(4): 431-9. doi: 10.1111/j.17441609.2008.00114.x.

22. Dakei Z, Hemmat Far A, Azizbeigi K. Effect of resistance and endurance training protocols on functional capacityand quality of life in male patients after myocardial infarction. Cardiovascular Nursing Journal. 2014; 3(1): 26-33.

23. Lee R, Chan YH, Wong J, Lau D, Ng K. The 6minute walk test predicts clinical outcome in Asian patients with chronic congestive heart failure on contemporary medical therapy: A study of the multiracial population in Singapore. Int J Cardiol. 2007; 119(2): 168-75.

24. Martin BJ, Arena R, Haykowsky M, Hauer T, Austford LD, Knudtson M, et al. Cardiovascular fitness and mortality after contemporary cardiac rehabilitation. Mayo Clin Proc. 2013; 88(5): 455-63. doi: 10.1016/j.mayocp.

25. American College of Sports Medicine. ACSM's Guidelines for Exercise Testing and Prescription. $6^{\text {th }}$ ed. Baltimore. Md: Lippincott Williams \& Wilkins. 2000.

26. Polock ML, Franklin B A, Balady GJ, chaitman BL, fleg JL, Fletcher $\mathrm{B}$, et al. Resistance Exercise In Individuals with and without cardiovascular Diseas. Circulation. 2000; 101: 828-833.

27. Enright PL. The six-minute walk test. Respiratory care. 2003 ; 48(8): 783-785.

28. Adams BJ, Carr JG, Ozonoff A, Lauer MS, Balady GJ. Effect of exercise training in supervised cardiac rehabilitation programs on prognostic variables from The exercise tolerance test. Am J Cardiol. 2008; 101(10): 1403-7. doi: 10.1016/j.amjcard.2008.01.016.

29. Choo J, Burke LE, Hong KP. Improved quality of life with cardiac rehabilitation for post-myocardial infarction patients in Korea. Eur J Cardiovasc Nurs. 2007; 6(3): 166-71.

30.Siavoshi S, Roshandel M, Zareiyan A, Ettefagh L. The effect of cardiac rehabilitation care plan on the quality of life in patients undergoing coronary artery bypass graft surgery. Cardiovascular Nursing Journal. 2012; 1(2): 38-46.
31. Ghroubi S, Elleuch W, Abid L, Abdenadher M, Kammoun S, Elleuch MH. Effects of a low-intensity dynamic-resistance training protocol using an isokinetic dynamometer on muscular strength and aerobic capacity after coronary artery bypass grafting. Ann Phys Rehabil Med. 2013; 56(2): 85-101. doi: 10.1016/j.rehab.2012.10.006.

32. Reibis RK, Treszl A, Wegscheider K, Ehrlich B, Dissmann R, Völler H. Exercise capacity is the most powerful predictor of 2-year mortality in patients with left ventricular systolic dysfunction. Herz. 2010; 35(2): 104-110. doi: 10.1007/s00059-010-3226-5.

33. Mampuya WM. Cardiac rehabilitation past, present and future: An overview. Cardiovasc Diagn Ther. 2012; 2(1): 38-49. doi: 10.3978/j.issn.2223-3652.2012.01.02.

34. Dusseldorp E, Van Elderen T, Maes S, Meulman J, kraaij V. A meta-analysis of psychoeducational programs for coronary heart disease patients. Health Psychology. 1999. 18(5): 506-19.

35. Beckie TM, Beckstead JW, Kip K, Fletcher G. Physiological and exercise capacity improvements in women completing cardiac rehabilitation. J Cardiopulm Rehabil Prev. 2013; 33(1):16-25. doi: 10.1097/HCR.0b013e3182763192.

36. Mozafari A, Hejazi SF, Baharvand A, Marvi M, Olomi Doran V, Mohebi S, Gharebeigloo M. The Effect of Cardiac Rehabilitation on the Results of 6-Minute Walk Test in Post Angioplasty Cardiac Patients Referred to Shahid Beheshti Hospital in Qom City, Iran. Qom Unive rsity of Medical Sciences Journal. 2015; 9(8): 4148.

37. Piepoli MF, Davos C, Francis DP, Coats AJ, ExTraMATCH Collaborative. Exercise training metaanalysis of trials in patients with chronic heart failure (ExTraMATCH). Bmj. 2004; 328(7433): 189.

38. Hwang CL, Chien CL, Wu YT. Resistance training increases 6-minute walk distance in people with chronic heart failure: a systematic review. J Physiother. 2010; 56(2): 87-96.

39. Sousa N, Mendes R, Abrantes C, Sampaio J, \& Oliveira J. A randomized 9-month study of blood pressure and body fat responses to aerobic training versus combined aerobic and resistance training in older men. Exp Gerontol. 2013; 48(8): 727-33. doi: 10.1016/j.exger.2013.04.008.

40. Fu, T. C., Wang, C. H., Lin, P. S., Hsu, C. C., Cherng, W. J., Huang, S. C., ... \& Wang, J. S. (2013). Aerobic interval training improves oxygen uptake efficiency by enhancing cerebral and muscular hemodynamics in patients with heart failure. International journal of cardiology, 167(1), 41-50.

41. Miranda MX, van Tits LJ, Lohmann C, Arsiwala T, Winnik S, Tailleux A, et al. The Sirt1 activator SRT3025 provides atheroprotection in Apoe-/- mice by reducing hepatic Pcsk9 secretion and enhancing Ldlr expression. Eur Heart J. 2015; 36(1): 51-9. doi: 10.1093/eurheartj/ehu095. 
42. Satoh A, Stein L, Imai S. The role of mammalian sirtuins in the regulation of metabolism, aging, and longevity, in Histone Deacetylases: the Biology and Clinical Implication. Springer. 2011; 125-162. doi: 10.1007/978-3-642-21631-2_7.

43. Russomanno G, Corbi G, Manzo V, Ferrara N, Rengo G, Puca AA, et al. The anti-ageing molecule sirt1 mediates beneficial effects of cardiac rehabilitation. Immun Ageing. 2017; 14: 7. doi: 10.1186/s12979-0170088-1.

44. Minamino T, Komuro I. Vascular cell senescence: contribution to atherosclerosis. Circ Res. 2007; 100(1): 15-26.

45. Saremi A, Sharjerdi S, Kavyani A. The effect of Aerobic Training on metabolic parameters and Serum level of sirtuinl in Women With type2.Diabetes.JArak unimed Sci. 2016; 19(9): 88-97.

46. Nara R, ScherolinO. Treadmill training increases SIRT1 and PGC-1 $\alpha$ protein levels and AMPK phosphorylation in quadriceps of middle-aged rats in an intensity-dependent manne. Mediators Inflamm. 2014; 2014: 1-11
47. Gurd BJ, Perry CG, Heigenhauser GJ, Spriet LL, Bonen A. High-intensity interval training increases SIRT1 activity in human skeletal muscle. Applied Physiology, Nutrition, and Metabolism. 2010; 35(3):3507.

48. Ma JK, Scribbans TD, Edgett BA, Boyd JC, Simpson $\mathrm{CA}$, Little JP, et al. Extremely low-volume, high-intensity interval training improves exercise capacity and increases mitochondrial protein content in human skeletal muscle. OJMIP. 2013; 3(04): 202-210. DOI: 10.4236/ojmip.2013.34027.

49. Marton O, Koltai E, Takeda M, Koch LG, Britton SL, Davies KJ, et al. Mitochondrial biogenesisassociated factors underlie the magnitude of response to aerobic endurance training in rats. Pflugers Arch. 2015 Apr;467(4):779-88. doi: 10.1007/s00424-014-1554-7. 